\title{
VORWORT ZUR RUSSISCHEN AUSGABE
}

Nach dem allgemeinen Plan dieses Lehrbuches ist der vorliegende Band der relativistischen Quantentheorie im weitesten Sinne dieses Wortes gewidmet; der Theorie aller Erscheinungen, bei denen die Endlichkeit der Lichtgeschwindigkeit eine Rolle spielt; dazu gehört auch die gesamte Strahlungstheorie.

Bekanntlich ist dieser Teil der theoretischen Physik gegenwärtig bei weitem noch nicht vollkommen ergründet; das gilt auch für die physikalischen Prinzipien der relativistischen Quantentheorie. Diese Feststellung bezieht sich insbesondere auf die Theorie der starken und der schwachen Wechselwirkungen. Aber selbst die Quantenelektrodynamik ist in ihrer logischen Struktur noch immer unbefriedigend, ungeachtet der in den letzten 20 Jahren erreichten blendenden Erfolge auf diesem Gebiet.

Bei der Stoffauswahl für dieses Buch haben wir uns auf diejenigen Ergebnisse beschränkt, die mit einem vernünftigen $\mathrm{MaB}$ an Sicherheit als genügend zuverlässig angesehen werden können. Bei einer solchen Auffassung beschäftigt sich natürlich der größte Teil des Buches mit der Quantenelektrodynamik. Wir haben uns bemüht, die Dinge von einem realistischen Standpunkt aus darzustellen und die in der Theorie angenommenen physikalischen Voraussetzungen hervorzuheben, ohne auf deren Begründung einzugehen. Eine solche Begründung hätte beim gegenwärtigen Stand der Theorie sowieso rein formalen Charakter.

Bei der Behandlung konkreter Anwendungen der Theorie haben wir uns nicht das Ziel gesteckt, die ungeheure Vielfalt der zugehörigen Effekte zu erfassen; wir haben uns auf die wesentlichen Effekte beschränkt und zusätzlich einige Hinweise auf Originalarbeiten gegeben, die eingehendere Untersuchungen enthalten. Die hier erforderlichen Rechnungen sind normalerweise sehr umfangreich. Wir haben daher häufig einige $Z$ wischenformeln weggelassen. Immer haben wir uns aber bemüht, alle verwendeten nichttrivialen methodischen Gesichtspunkte anzugeben.

Gegenüber den anderen Bänden dieses Lehrbuches setzt dieses Buch ein höheres Niveau des Lesers voraus. Wir sind davon ausgegangen, daß ein Leser, der beim Studium der theoretischen Physik die Quan- 
tenfeldtheorie erreicht hat, keine überflüssigen Erläuterungen des Stoffes mehr benötigt.

Die Aufteilung des Buches in zwei Teile ist nicht von prinzipieller Natur, sie hängt lediglich mit dem großen Stoffumfang zusammen. Der zweite Teil enthält die Strahlungskorrekturen in der Quantenelektrodynamik, die Theorie der schwachen Wechselwirkungen und einige Probleme aus der Theorie der starken Wechselwirkungen.

Dieses Buch ist ohne direkte Beteiligung unseres Lehrers L. D. LANDAU geschrieben worden. Wir sind aber immer bemüht gewesen, uns von demselben Geist und von demselben Verhältnis zur theoretischen Physik leiten zu lassen, das er uns gelehrt hat und das er auch in den anderen Bänden dieses Lehrbuches eingenommen hat. Wir haben uns häufig selbst gefragt, wie sich wohl DAU zu dieser oder jener Frage verhalten würde, und wir haben uns bemüht, so zu antworten, wie es uns die jahrelange Gemeinsamkeit mit ihm eingegeben hat.

Wir danken W. N. Barer für die große Hilfe bei der Zusammenstellung von $\S 59$ und 94 sowie W. I. RITus für die große Hilfe beim Schreiben von $\S 98$. Auch sind wir B. E. MeIERowitsch für die Hilfe bei einigen Berechnungen zu Dank verpflichtet. Ferner möchten wir A. S. Kompanejez für die Überlassung seiner Nachschrift der Vorlesung über Quantenelektrodynamik, die L. D. LANDAU 1959/60 an der Moskauer Universität gehalten hat, unseren Dank aussprechen.

Dieses Buch ist das Ergebnis einer Gemeinschaftsarbeit; sein Inhalt wurde von allen Verfassern gemeinsam erörtert. Im einzelnen wurde die Arbeit folgendermaßen verteilt: Die Kapitel I und V wurden von E. M. Lifschitz geschrieben, die Kapitel II, III und VII stammen von W. B. BerestezkI und E. M. Lifschitz, die Kapitel IV, VI und IX von E. M. Lifschitz und L. P. Pitajewski, und die Kapitel VIII und $\mathrm{X}$ wurden von allen drei Autoren gemeinsam verfaßt.

Juni 1967

W. B. BeRESTEzKI

E. M. Lifschitz

L. P. Pitajewski 